\title{
First experimental studies of the physics of plasmas of arbitrary degree of neutrality
}

\author{
X Sarasola and T Sunn Pedersen \\ Max-Planck-Institut für Plasmaphysik, EURATOM Association, Teilinstitut Greifswald, \\ Wendelsteinstr. 1, 17491 Greifswald, Germany \\ Dept. of Applied Physics and Applied Mathematics, Columbia University, New York, NY \\ 10027, USA \\ E-mail: xabier.sarasola@ipp.mpg.de
}

\begin{abstract}
The first detailed experimental characterization of the physics of plasmas of arbitrary neutrality is reported. It is shown that the degree of neutralization of the plasma in the Columbia Non-Neutral Torus stellarator can be varied continuously from quasi-neutral to pure electron plasma, and that the plasma can be sustained indefinitely at any degree of neutralization. The physical phenomena change significantly as the degree of neutralization is varied. Quasi-neutral plasmas exhibit a single mode low frequency flute instability. This mode aligns almost perfectly with the magnetic field lines, presenting a resonant $m=3$ poloidal structure. For weakly magnetized ions, and moderate degrees of neutralization, multi-mode behaviour is observed, in some cases fully turbulent. At the other extreme of the neutralization scale, electron-rich plasmas with a small but non-negligible amount of ions exhibit single mode fluctuations. Essentially identical phenomena were previously reported by Marksteiner [Q. Marksteiner et al., 2008, Phys. Rev. Lett., 100, 065002]. In addition to the already known poloidal mode number $m=1$ of these fluctuations, the toroidal mode number is now known: $n=0$. This not only confirms the previous observations that the electron fluid force balance appears to be broken, but the frozen-in flux condition is also violated.
\end{abstract}

\section{Introduction}

The physics of partially neutralized plasmas remains largely unexplored, perhaps because of the difficulty of confining such plasmas and/or finding experimental conditions where the plasma does not quickly convert to the quasi-neutral state. Penning-Malmberg traps $[1,2]$ confine plasmas of one sign of charge only, but cannot confine partly neutralized or quasi-neutral plasmas. Tokamaks, on the other hand, require large internal currents for confinement, which cannot be driven in the low densities typical of non-neutral plasmas [3,4]. Pure toroidal traps $[5,6]$ have been successfully used to confine non-neutral plasmas, and have also been used to study quasi-neutral plasmas [7], but have to date not yet confined and sustained partially neutralized plasmas. Stellarators, exhibiting closed particle orbits at any degree of neutralization, are predicted to be able to confine plasmas of arbitrary neutrality $[8,9]$. This paper confirms that it is experimentally possible to create, sustain, and study plasmas at any degree of neutralization in a stellarator. The physics of plasmas confined in the Columbia NonNeutral Torus (CNT) stellarator is investigated as the degree of neutrality is varied continuously all the way from quasi-neutral to pure electron. 


\section{Basic experimental setup}

CNT is a university-scale, two period, classical stellarator in which the magnetic surfaces are created by just two pairs of circular planar coils [10]. The rotational transform $(\iota)$ describes the poloidal twist of the field lines around the torus in a magnetic surface configuration [11]. In CNT, the shape and the $\iota$-profile of the magnetic surfaces is mainly determined by the tilt angle between the two internal coils (adjustable in three positions: $64^{\circ}, 78^{\circ}$ and $88^{\circ}[12]$ ).

Pure electron plasmas have been studied in detail in CNT in the past [13-16]. A typical $\phi_{\text {plasma }}=-200 \mathrm{~V}$ pure electron plasma is characterized by a flat temperature profile with $T_{\mathrm{e}}$ between 2 and $7 \mathrm{eV}$, and a density profile peaked off-axis with $n_{\mathrm{e}} \approx 3 \times 10^{12} \mathrm{~m}^{-3}$. These measurements imply that many Debye lengths of pure-electron plasma are confined in CNT: $\lambda_{\mathrm{D}} /\langle a\rangle=0.1$, with $\langle a\rangle=15 \mathrm{~cm}$ being the average minor radius of the plasma.

Steady state plasmas are routinely created by thermionic emission from a filament located close to the magnetic axis [17]. Particle flux probes are used for equilibrium measurements [14], and are mounted on ceramic rods held inside the plasma. These rods charge up negatively and self-shield against the otherwise large electron flux. These negative rods act as sinks for the ions, which are created through volumetric ionization of neutral gas, and a steady-state ion content proportional to neutral pressure results [18]. Thus, the degree of non-neutrality is controllable in CNT. We parameterize the degree of non-neutralization by the dimensionless quantity $\eta$ :

$$
\eta \equiv\left|\frac{n_{\mathrm{e}}-Z n_{\mathrm{i}}}{n_{\mathrm{e}}+Z n_{\mathrm{i}}}\right|
$$

In CNT, $\eta$ has now been varied continuously from $\eta<10^{-4}$ (a quasi-neutral plasma), to $\eta=1$ (a pure electron plasma), simply by adjusting the neutral pressure in the chamber, figure 1 . It is possible to operate continuously at any point on the curve shown.

Even though the electron density measurements in CNT have very large uncertainties [14], $\eta$ has been determined experimentally within about $30 \%$ (and is known within $6 \%$ for $\eta \approx 1$ ) without measuring $n_{\mathrm{e}}$ directly. The ion density (or more specifically, $Z n_{\mathrm{i}}$ ) is determined from a measurement of the ion saturation current collected from a $7.4 \mathrm{~cm}^{2}$ copper plate inserted at $\psi \approx 0.4[18]$ ( $\psi$ is the magnetic surface coordinate [19]). The numerator in $\eta$ (space charge) can be determined with good accuracy using measurements of the space potential $\phi$, combined with Poisson's equation in CNT's complicated 3D geometry [20], $\frac{\epsilon_{0}}{e} \nabla^{2} \phi=n_{\mathrm{e}}-Z n_{\mathrm{i}}$. This method is sufficiently accurate for our purpose since we vary $\eta$ by four orders of magnitude here.

\section{Overall behaviour}

Fluctuations in the plasma potential $(\widetilde{\phi})$ are detected by measuring the oscillating current in an internal floating emissive (hot) filament [21]. Perturbations in the plasma were also measured with an ion probe, a set of capacitive probes, and a high-speed camera. The signals recorded in these diagnostics are very well correlated with $\widetilde{\phi}$.

Figure 2 shows the amplitude of the fluctuations in a floating emissive probe, and the average charge loss rate as $\eta$ is swept from quasi-neutral to pure electron. Confinement times are short, and low frequency spontaneous oscillations are observed for quasi-neutral plasmas confined in CNT. The amplitude of the detected fluctuations reaches a maximum for $\eta \approx 10^{-2}$, but even at the highest amplitudes, the observed oscillatory perturbations satisfy $|e \widetilde{\phi}| / T_{\mathrm{e}}<1$. Confinement of pure electron plasmas $(\eta \approx 1)$ is enhanced by the strong radial electric field created by the large space charge. These plasmas are stable, quiescent, and have been extensively studied in CNT $[13,15]$.

The plasma potential $\left(\phi_{\mathrm{p}}\right)$ has been determined using two methods: measuring the deviation potential $\left(\phi_{\mathrm{dev}}\right.$, potential at which a heated filament becomes slightly more negative than the local plasma potential and begins to emit electrons, [14]), and measuring the voltage required 


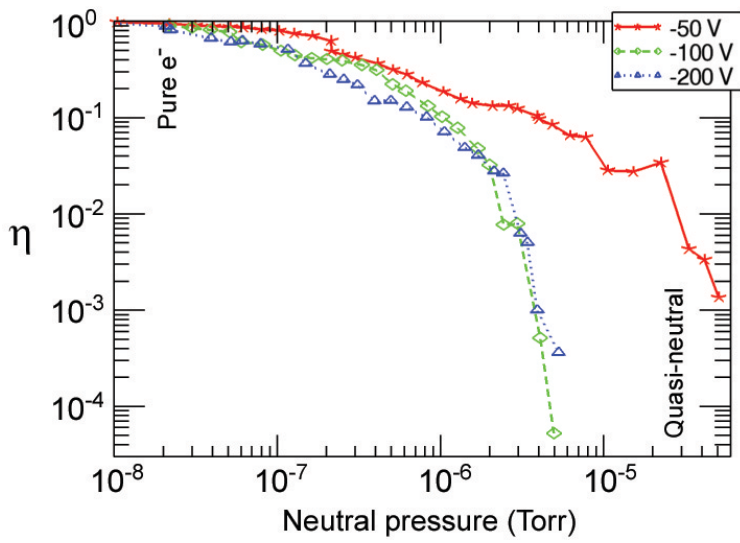

Figure 1. Degree of non-neutralization $(\eta)$ as a function of the neutral pressure in the chamber for different emitter bias voltages $\left(\mathrm{N}_{2}^{+}-e^{-}\right.$plasmas at $\left.0.02 \mathrm{~T}\right)$.

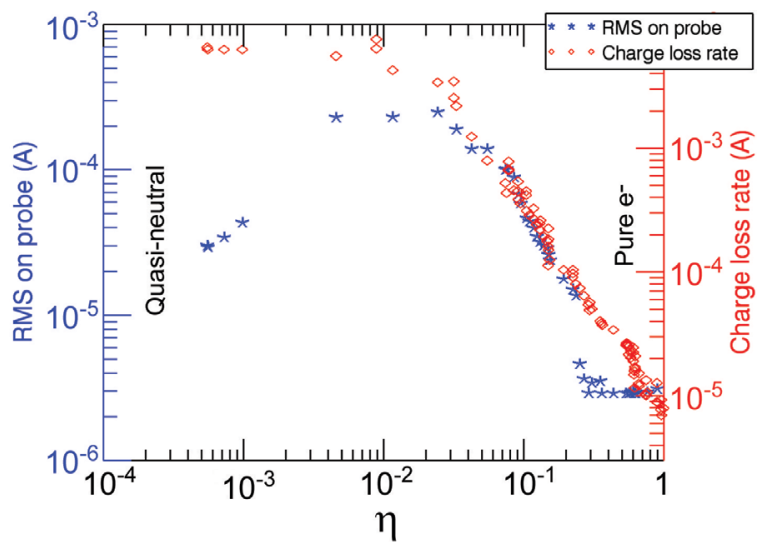

Figure 2. Charge loss rate and RMS of the oscillations in a floating emissive probe as the degree of non-neutralization is varied $\left(\mathrm{N}_{2}^{+}-e^{-}\right.$ plasmas, $\left.\mathrm{B}=0.02 \mathrm{~T}, \phi_{\mathrm{e}}=-200 \mathrm{~V}\right)$.

to float an emissive filament $\left(\phi_{\mathrm{fe}}\right)$ inserted in the plasma. $\phi_{\mathrm{fe}}$ floats close to $\phi_{\mathrm{p}}$ in cold plasmas:

$$
\phi_{\mathrm{fe}}=\phi_{\mathrm{p}}-\alpha \frac{T_{\mathrm{e}}}{e}
$$

where $\alpha \approx 1$ for $\mathrm{N}_{2}^{+}-e^{-}$plasmas [22]. Pure electron plasmas are characterized by a close match between the plasma potential on axis and the emitter filament bias voltage creating the plasma $\left(\phi_{\mathrm{e}}\right)$. The zero potential boundary is located at the chamber walls. The addition of ions (lowering of $\eta$ ) alters electron-rich plasmas. For $\eta<0.3, \phi_{\mathrm{p}}$ begins to decouple from $\phi_{\mathrm{e}}$ (figure 3(a)), and spontaneous fluctuations appear (figure 2). For $\phi_{\mathrm{e}}=-200 \mathrm{~V}$, the plasma potential of a quasi-neutral plasma $\left(\eta \approx 10^{-4}\right)$ is completely decoupled, $\phi_{\mathrm{p}} \approx-8 \mathrm{~V}$.

$T_{\mathrm{e}}$ in electron-rich plasmas has been measured by interpreting the I-V characteristics of an internal Langmuir probe (from the slope of the linear part of the plot $\log \left(I_{\text {probe }}\right)$ vs $\left.\phi_{\text {probe }}-\phi_{\mathrm{p}},[23]\right)$. Figure 3(b) shows how the electron fluid becomes hotter as the plasma detaches from the emitter bias voltage. This comes about because the electrons gain kinetic energy when accelerating away from the $-200 \mathrm{~V}$ emitter filament to the plasma.

We attempted to use the same method to measure $T_{\mathrm{e}}$ also for quasi-neutral plasmas. Since in CNT electrons are magnetized, and the ions are unmagnetized compared to the sheath size, the measured electron temperature from the slope of the plot $\log \left(I_{\text {probe }}\right)$ vs $\phi_{\text {probe }}-\phi_{\mathrm{p}}$ should agree with the textbook equation [23],

$$
\phi_{\mathrm{f}}=\phi_{\mathrm{p}}-\frac{T_{\mathrm{e}}}{2 e}\left(1+\ln \left(\frac{m_{\mathrm{i}}}{2 \pi m_{\mathrm{e}}}\right)\right)
$$

Direct measurements of the cold floating potential $\left(\phi_{\mathrm{f}}\right)$ and the deviation potential $\left(\phi_{\text {dev }}\right)$ indicate that $\phi_{\mathrm{f}} \approx-20 \mathrm{~V}$, and $\phi_{\mathrm{p}}=\phi_{\mathrm{dev}} \approx-8 \mathrm{~V}$ in the quasi-neutral regime $\left(\eta \approx 10^{-4}\right)$. This implies that the electron temperature is $T_{\mathrm{e}} \approx 2 \mathrm{eV}$, which is also in agreement with equation 2 (see figure 4 for further explanations). However, the estimated electron temperature obtained from the slope of the $\log \left(I_{\text {probe }}\right)$ vs $\phi_{\text {probe }}-\phi_{\mathrm{p}}$ plot is $T_{\mathrm{e}} \approx 50 \mathrm{eV}$. Such a large value of $T_{\mathrm{e}}$ is unphysical for our plasmas, and is likely caused by the combination of the lack of a good separation between the ion saturation regime and the onset of electron saturation in the I-V curves. 
The ion temperature has not been measured. However, one can provide a rough estimate as follows: First note, that no direct ion heating is applied in CNT, and that ions are born through ionization near room temperature. We assume that the only source of ion acceleration/heating is the space electric field. For unmagnetized ions, the ion kinetic energy will average about half of the potential energy they have at the center of the electron rich (negatively biased) plasma. For magnetized ions, only the initial acceleration before completion of the Larmor orbit will cause a net energy gain - the ions will then $E \times B$ drift and gyrate subsequently. Generically, that leads to an ion velocity on the order of the $E \times B$ drift velocity. For $\eta=1$ and $\phi_{\mathrm{p}}=-200$ $\mathrm{V}$ in the center of the plasma, massive ions (e.g., $\mathrm{N}_{2}^{+}$) are largely unmagnetized, and have an average kinetic energy that varies up to $200 \mathrm{eV}$, as they bounce through the electron cloud. For $\eta \approx 10^{-4}$ and $\phi_{\mathrm{p}} \approx-8 \mathrm{~V}$, the ions do not accelerate much, they stay well magnetized, and their velocities approach the $E \times B$ velocity (for a detailed numerical study of various ion orbits in CNT, see ref. [24]). In this case, the estimated $\mathrm{N}_{2}^{+}$gyro-radius is $r_{\mathrm{Li}}=m_{i} v_{E \times B} / q B \approx 2 \mathrm{~cm}$ and the estimated ion temperature is $T_{\mathrm{i}} \approx 0.25 \mathrm{eV}$, for $\eta \approx 10^{-4}$ and $\mathrm{B}=0.02 \mathrm{~T}$.
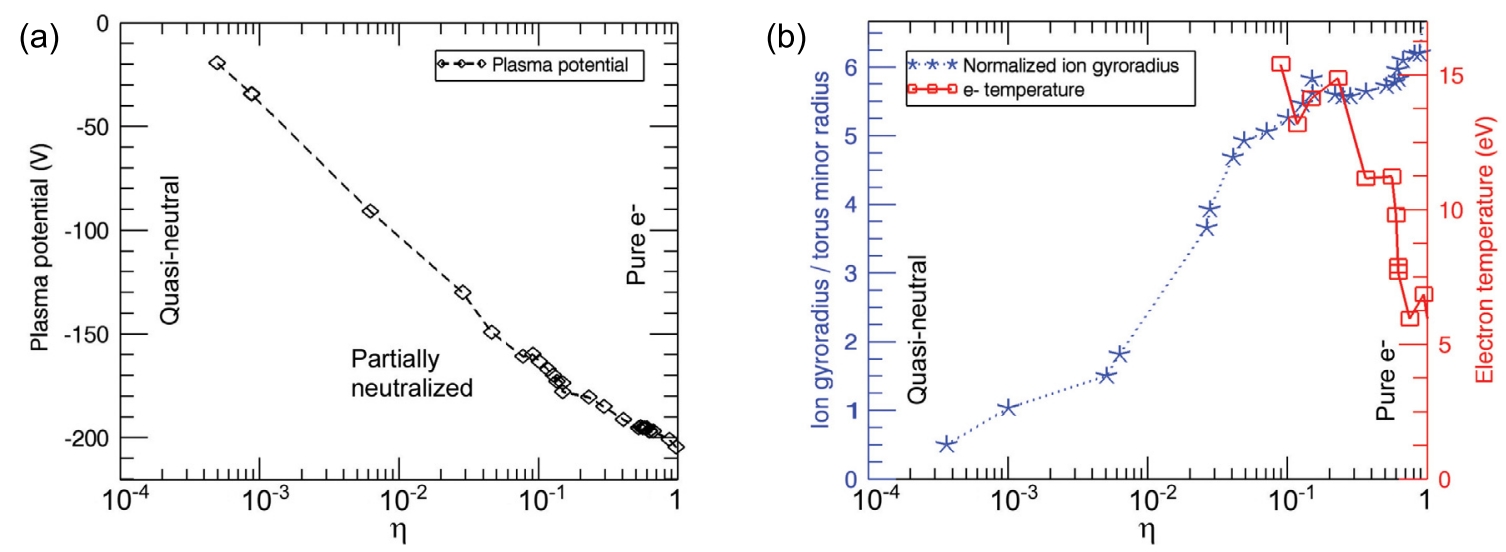

Figure 3. (a) Plasma potential at $\psi \approx 0.1$ vs. degree of non-neutralization $(\eta)$ while emitting electrons on the magnetic axis at $-200 \mathrm{~V}$. (b) Measured electron temperature compared to the normalized ion gyro-radius as a function of $\eta\left(\mathrm{N}_{2}^{+}-e^{-}\right.$plasmas at $\left.\mathrm{B}=0.02 \mathrm{~T}\right)$.

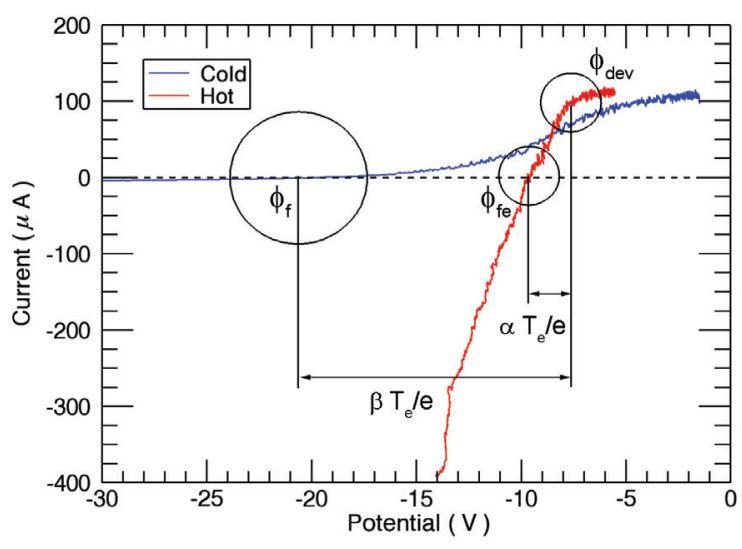

Figure 4. Hot and cold $\mathrm{I}-\mathrm{V}$ characteristics of a probe located at $\psi \approx 0.1$. $\phi_{\mathrm{f}}$ is the floating potential of the cold probe, $\phi_{\mathrm{fe}}$ refers to the floating potential of the emissive (hot) probe, and $\phi_{\mathrm{dev}}$ is the deviation potential. The circles indicate the measurement error. $\alpha \approx 1$ for a $\mathrm{N}_{2}^{+}-e^{-}$plasma [22], and $\beta=\frac{1}{2}\left(1+\ln \left[m_{\mathrm{i}} /\left(2 \pi m_{\mathrm{e}}\right)\right]\right)$. Hot characteristics were sometimes affected by a $4 \mathrm{~V}$ offset due to the polarity of the battery used to heat the filament probe. The hot characteristic shown here is corrected for this. Emitter bias $\phi_{\mathrm{e}}=-200 \mathrm{~V}, \mathrm{~B}=0.08 \mathrm{~T}$, and $\eta \approx 10^{-4}$. 


\section{Quasi-neutral plasmas}

Quasi-neutral plasmas in CNT $\left(\eta \lesssim 10^{-4}\right)$ are characterized by weak electric fields $\left(\left|e \phi_{\mathrm{p}}\right| / T_{\mathrm{e}} \approx\right.$ 1 ), and the presence of spontaneous single mode low frequency oscillations $(1-20 \mathrm{kHz})$ for all magnetic field strengths studied. The measured frequency of the mode has a negligible dependence on $\eta$. Figure 5 plots the mode frequency vs the ratio $-\phi_{\mathrm{p}} / B$. The mode appears to have an approximately offset linear relationship with $E / B$. The offset is believed to be due to the accuracy $\left(\sim T_{\mathrm{e}} / e\right)$ in the determination of $\phi_{\mathrm{p}}$ using a floating emissive probe.

The frequency of the oscillations has a flat profile across the plasma, showing that the detected instability is a global mode (the plasma moves as a whole), and no velocity shear is observed.

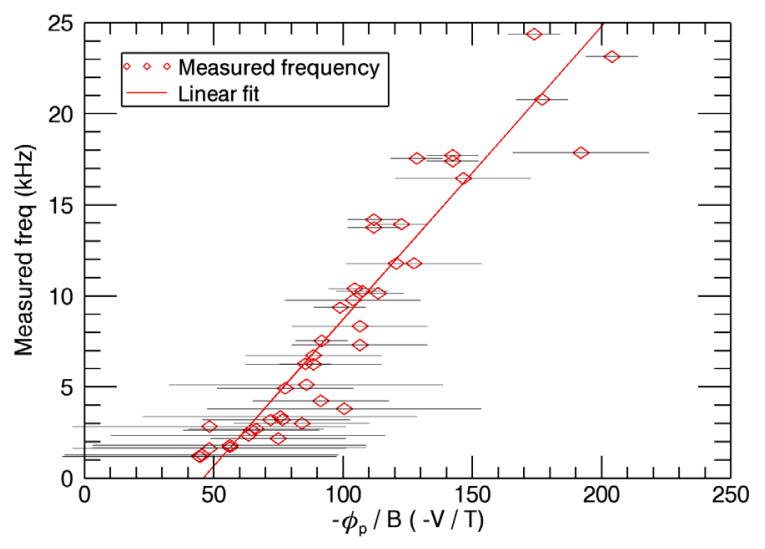

Figure 5. Measured frequency of the dominant mode in quasi-neutral plasmas vs $-\phi_{\mathrm{p}} / B . \mathrm{N}_{2}^{+}-e^{-}$plasmas, $0.02 \leq B \leq 0.10$ $\mathrm{T},-400 \leq \phi_{\mathrm{e}} \leq-100 \mathrm{~V}$.

\subsection{Local $\widetilde{n}-\widetilde{\phi}$ phase shift}

The local phase shift between density and potential fluctuations was measured using a relatively simple and well-established method $[25,26]$ which involves the use of two probes, one of them working as a reference. Two independent sets of experiments were run to measure respectively $-\widetilde{\mathrm{I}}_{\text {sat }}$ and $\widetilde{\phi}$ at one location, and a nearby probe measured $\widetilde{\phi}$ in both experiments and was used as the reference. If the oscillations in $T_{\mathrm{e}}$ are assumed to be small, the phase difference between $-\widetilde{\mathrm{I}}_{\mathrm{sat}}$ and $\widetilde{\phi}_{\mathrm{f}}$ is a reasonably good estimator of the phase shift between $\widetilde{n}$ and $\widetilde{\phi}$. For quasi-neutral plasmas, $\widetilde{n}$ leads $\widetilde{\phi}$ by $34^{\circ} \pm 57^{\circ}$, which, based on the criteria derived by Jassby [27], rules out Kelvin-Helmholtz instabilities and slightly favors drift waves over Rayleigh-Taylor oscillations. However, the large uncertainty in the determination of the phase difference does not allow us to clearly distinguish between drift waves and a Rayleigh-Taylor instability based on this criterion only. This prompted the development of a new method to simultaneously measure the oscillations in $n$ and $\phi$, described in the following.

The fluctuations were recorded in a single Langmuir probe as the probe bias voltage was slowly varied $(\sim 40 \mathrm{~V} / \mathrm{s})$ between $\phi_{\mathrm{p}}$ and $\phi_{\mathrm{p}}-100 \mathrm{~V}$. The Empirical Mode Decomposition method [28] was applied to extract the dominant mode of the probe signal, and the signal was binned at multiple phase-locked bins. Four phase bins are coloured in figure 6(a), and the corresponding I-V characteristics are plotted in figure 6(b). Once the different phase-locked I-V curves are extracted, the plasma density $(n)$, and floating potential $\left(\phi_{\mathrm{f}}\right)$ can be determined selfconsistently for each I-V trace [23]. The diamonds and crosses in figure 6(b) show respectively the ion saturation current (at $\phi_{\text {probe }}=-100 \mathrm{~V}$ ) and the floating potential for 4 phase-locked I-V curves. $-\widetilde{I}_{\text {sat }}$ and $\widetilde{\phi}_{\mathrm{f}}$ are always very close to being in phase, which provides more arguments for explaining this mode as drift waves. In principle, $T_{\mathrm{e}}$ can also be obtained by interpreting the slope of each I-V trace, but this method does not produce good estimates for $T_{\mathrm{e}}$ in our quasi-neutral plasmas (see section 3). 

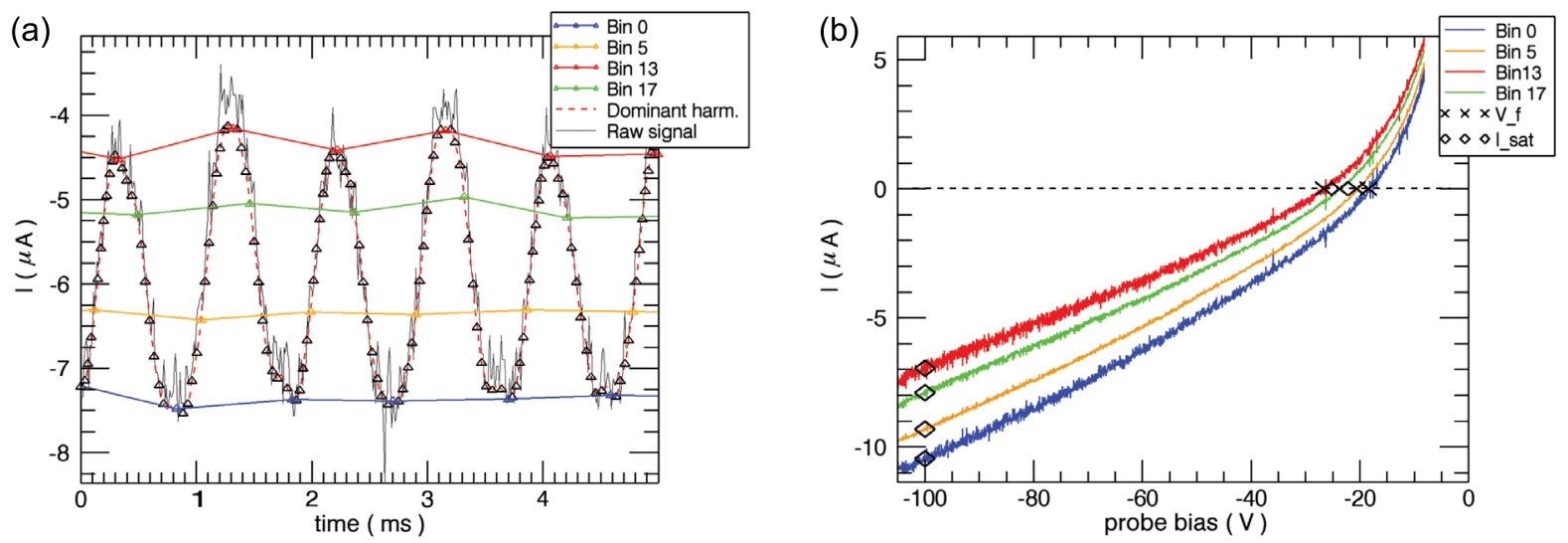

Figure 6. (a) Measured current to the Langmuir probe vs. time. $\triangle$ are samples of 25 phaselocked bins overlaid (the 4 coloured phase bins correspond to the I-V curves shown in (b)). (b) Langmuir I-V characteristics of 4 different phase bins. $\diamond$ and $\times$ show respectively the ion saturation current and floating potential of each I-V characteristic. Quasi-neutral plasma at $\mathrm{p}_{\mathrm{n}}=2.0 \times 10^{-5}$ Torr of $\mathrm{N}_{2}, \phi_{\mathrm{e}}=-200 \mathrm{~V}, \mathrm{~B}=0.02 \mathrm{~T}$.

\subsection{Spatial Structure}

The spatial structure of the mode was studied in detail in the $78^{\circ}$ configuration with a high speed camera. The detected instability propagates in the $\boldsymbol{E} \times \boldsymbol{B}$ direction. The magnetic field lines were also recorded with the same camera to study the alignment of the mode, using a field visualization technique described in previous publications [10,29]. This method uses an internal filament to emit an electron beam while the volume of the magnetic surfaces is intersected with a grounded aluminum rod to prevent the formation of the plasma. The luminescent trace of the e-beam is recorded as it follows the magnetic field lines, and the brightest pixels of each trace are stored for comparison with movies of the plasma fluctuations. Figures $7(\mathrm{a})$ and $7(\mathrm{~b})$ compare phase plots of the oscillations with traces of the field lines as seen from two view ports: the side window looks radially into the plasma, and the top window looks perpendicularly at the $\varphi=90^{\circ}$ cross-section of the torus. The instability is a flute-like mode (i.e., $\boldsymbol{k} \cdot \boldsymbol{B} \approx 0$ ).

Measurements with an array of capacitive probes around the plasma show a $m=3$ poloidal mode structure. Moreover, since the mode is closely aligned with B in the view of the camera, and the rotational transform is near or at $\iota=1 / 3$ across the magnetic surfaces in this $\left(78^{\circ}\right)$ configuration, this is clearly a resonant $\iota=n / m=1 / 3$ mode. The video diagnostic was also used to confirm this result. Movies of the oscillations from the "top view" port were compared with simulated movies of $m=1,2,3$, and 4 modes propagating in the torus, and the simulated $m=3$ phase plot (figure $7(\mathrm{e})$ ) provides the best match.

\subsection{Discussion}

Table 1 compares features of CNT's flute instability with predicted values for drift waves (driven by density gradients) and Rayleigh-Taylor instabilities (driven by magnetic curvature) using the criteria derived by Jassby [27]. The modes are best described as drift waves. The mode rotation frequency is close to $\omega_{E \times B}$, but it is difficult to conclude whether the measured frequency is $\omega_{E \times B}$ or $\omega_{E \times B} \pm \omega_{*}$, since $\omega_{* \mathrm{i}} \ll \omega_{* \mathrm{e}}<\omega_{E \times B}$. 


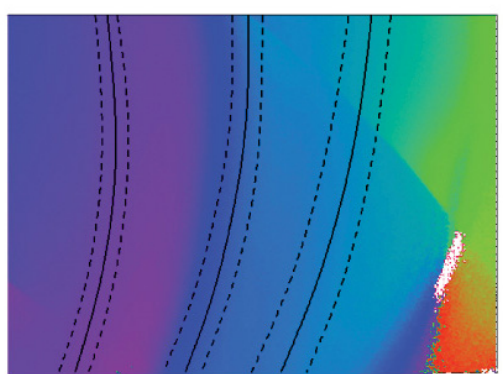

(a) Side view - Experimental

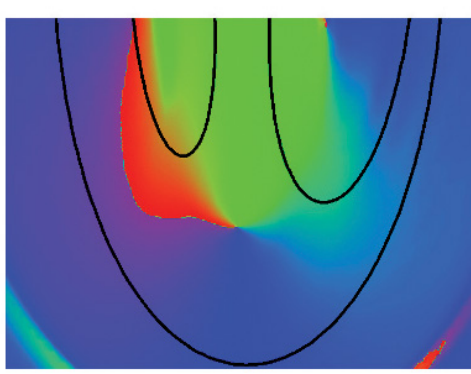

(c) Top view - Simulated $m=1$

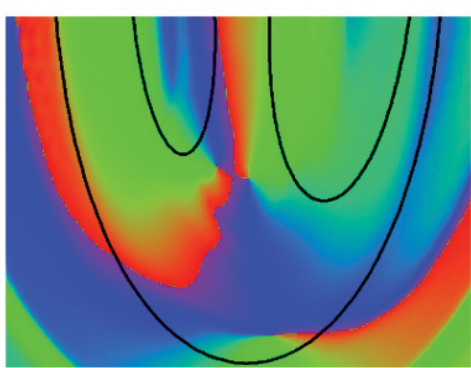

(e) Top view - Simulated $m=3$
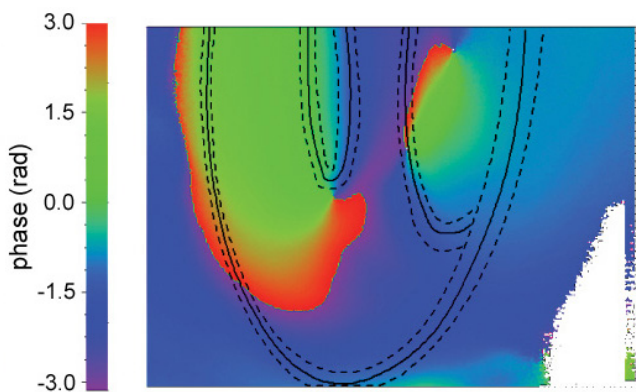

(b) Top view - Experimental
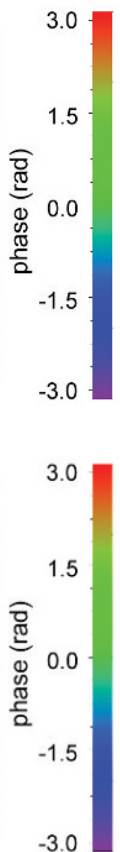

$-3.0$

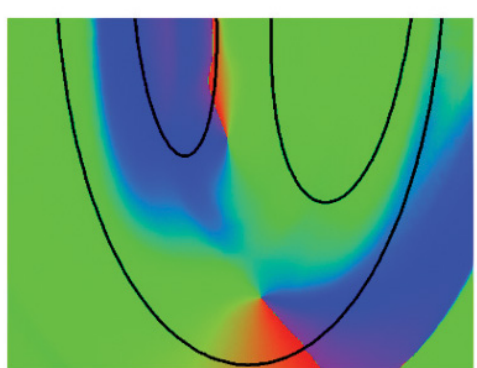

(d) Top view - Simulated $\mathrm{m}=2$

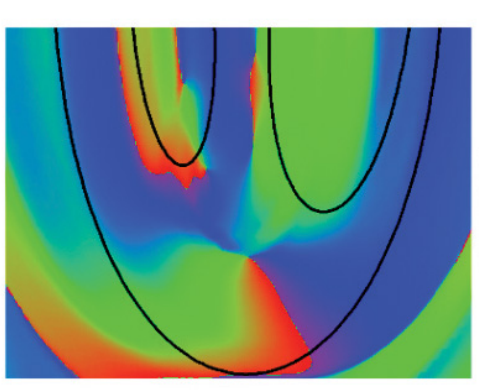

(f) Top view - Simulated $m=4$
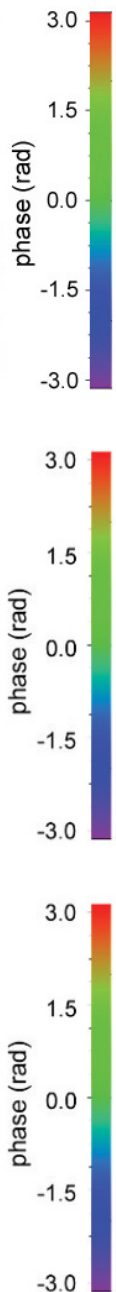

$-3.0$

Figure 7. Phase plots of the dominant mode of the oscillations in $\eta \approx 10^{-4}$ plasmas. Traces of the field lines are overlaid in solid black with dashed lines showing the error bars. Non-oscillating pixels are plotted in white. (a) Phase plot of a movie recorded from the side port of the chamber. (b) Phase plot of a movie taken from the top window of the chamber. (c), (d), (e) and (f) are respectively phase plots of the simulated $m=1,2,3$ and 4 modes as seen from the top window of the chamber.

\section{Partially neutralized plasmas}

\subsection{Temporal behaviour}

Low frequency modes $\left(\omega \ll \omega_{\mathrm{p}}\right)$ are predicted to be stable in pure electron plasmas confined by magnetic surfaces [8]. In agreement with this, we generally observe quiescent behaviour when the ion content is low $(\eta>0.9)$. Spontaneous broad-band fluctuations appear when a certain ion content is exceeded $(\eta \lesssim 0.8)$. Largely unmagnetized ions bounce chaotically in electron-rich partially neutralized plasmas $\left(10^{-4}<\eta<0.8\right)$ and multiple modes are excited.

Figure 8 illustrates how the power spectrum of the oscillations narrows down as the B field strength is increased. When more magnetized ions are present in the plasma (either by introducing lighter ion species $\left(\mathrm{He}^{+}\right)$or increasing the $\mathrm{B}$ field), the plasma reverts to single mode behaviour in the range $0.03<\eta<0.8$. This single mode was previously discovered and 
Table 1. Comparison of experimental characteristics of CNT's quasi-neutral mode with predicted features for drift waves and Rayleigh-Taylor instabilities [27].

\begin{tabular}{|c|c|c|c|c|}
\hline & & $\mathrm{CNT}$ & Drift waves & Rayleigh-Taylor \\
\hline frequency & & $\sim \omega_{E \times B}$ & $\omega_{E \times B} \pm \omega_{*}$ & $\omega_{E \times B}$ \\
\hline \multirow[t]{2}{*}{$\widetilde{n}-\widetilde{\phi}$ phase shift: } & 2 -probe method & $34^{\circ} \pm 57^{\circ}$ & $0^{\circ}$ to $45^{\circ}$ & $45^{\circ}$ to $90^{\circ}$ \\
\hline & 1-probe method & $0^{\circ} \pm 2^{\circ}$ & $0^{\circ}$ to $45^{\circ}$ & $45^{\circ}$ to $90^{\circ}$ \\
\hline$k_{\|}$ & & $\sim 0$ & $>0$ & 0 \\
\hline mode number & & 3 & no restriction & 1 or $2^{\mathrm{a}}$ \\
\hline$\left|e \widetilde{\phi} / T_{\mathrm{e}}\right| /|\widetilde{n} / n|$ & & usually $<1$ & $\lesssim 1$ & $\gtrsim 1$ \\
\hline
\end{tabular}

${ }^{\mathrm{a}}$ In the absence of magnetic surfaces.

studied in CNT's standard configuration (64 tilt angle) and has been identified as an ion-driven instability [21]. In the standard configuration, the rotational transform of the magnetic surfaces varied from $\iota=0.12$ on the magnetic axis to 0.23 at the plasma edge [12]. The tilt angle between the CNT's interlocking coils has since been switched to $78^{\circ}$. In this new configuration CNT has an almost flat $\iota$-profile $(\iota \approx 1 / 3)[12]$. However, despite the substantial difference in the magnetic topology, the behaviour of the ion-driven mode is essentially the same. The frequency of the mode is in the range between 20 and $100 \mathrm{kHz}$, scales close to linear with $E / B$, and presents a small dependence on the ion species introduced. The density and potential fluctuations driven by the ion-instability are in phase within the measurement uncertainty.

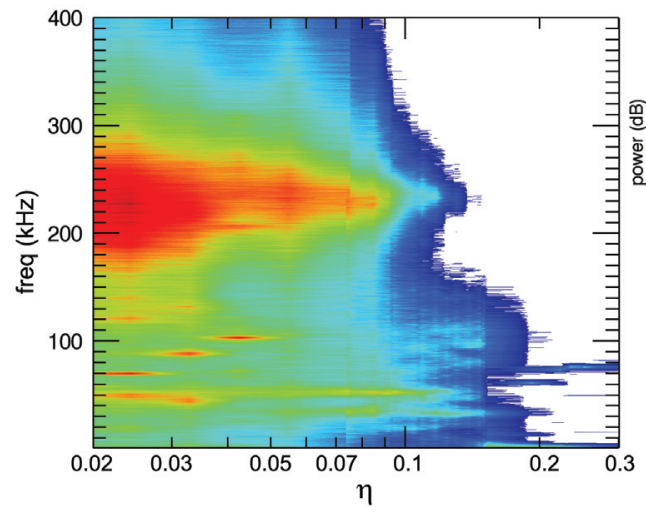

(a) $\mathrm{B}=0.02 \mathrm{~T}$.

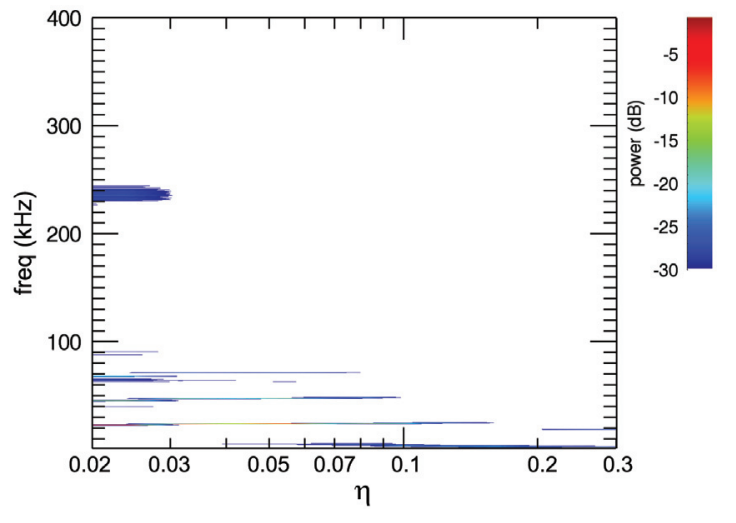

(b) $\mathrm{B}=0.08 \mathrm{~T}$.

Figure 8. Power spectrum of the signal on a floating emissive probe vs $\eta . \mathrm{N}_{2}^{+}-e^{-}$plasmas, $\phi_{\mathrm{e}}=-200 \mathrm{~V}$.

\subsection{Spatial Structure}

The spatial structure of the ion mode has been studied for the $78^{\circ}$ tilt angle with two perpendicular arrays of capacitive probes around the poloidal and toroidal angle of the plasma. The mode travels in the $\boldsymbol{E} \times \boldsymbol{B}$ direction and the poloidal mode number is $m=1$ (as was the case in the $64^{\circ}$ configuration [21]). The ion-related mode does not align itself to the field even with this low shear rotational profile, instead again chooses a $m=1$ poloidal mode number.

The toroidal mode number has now also been measured. It was expected to be $n>0$, in order to conserve the poloidal magnetic flux in the stellarator, but figure 9 shows that the mode 

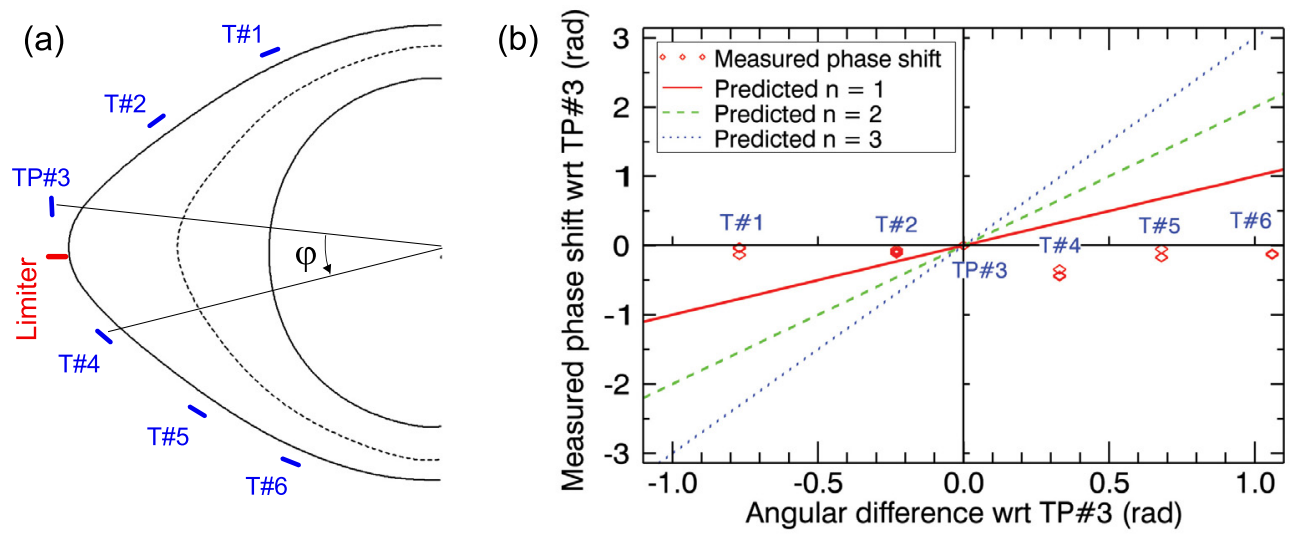

Figure 9. (a) Layout of the capacitive probe array around the toroidal cross-section of CNT (the dashed line is a projection of the magnetic axis). (b) Measured phase shift of the ion-driven mode detected on the toroidal capacitive probes. The phase on probe TP $\sharp 3$ is used as the reference. $\mathrm{p}_{\mathrm{n}}=4 \times 10^{-7}$ Torr of $\mathrm{N}_{2}, \phi_{\mathrm{e}}=-200 \mathrm{~V}, \mathrm{~B}=0.08 \mathrm{~T}$.

is $n=0$, a finding that was confirmed by the measurement in an additional capacitive probe installed $180^{\circ}$ toroidally displaced from probe $\mathrm{TP} \sharp 3$.

\subsection{Discussion}

The most significant difference between the results presented here and previously published observations [21] is the presence of two rather different regimes of oscillations in partially neutralized plasmas: 1) the single mode ion-driven instability, and 2) multi-mode / broadband oscillations. Single mode oscillations are now detected only for $0.03<\eta<0.8$ at high B field strengths $(\mathrm{B}>0.04 \mathrm{~T})$ or when light ion species $\left(\mathrm{He}^{+}\right)$are present in the electron-rich plasma. Hence, the presence of magnetized ions traveling close to the $E \times B$ rotation of the plasma seems to play an essential role in the excitation of CNT's single mode ion-resonant oscillations. Otherwise, broadband fluctuations are observed. Plasmas with degrees of non-neutrality between $10^{-4}$ and 0.03 are always characterized by the presence of broadband oscillations and a rather abrupt decoupling of the plasma potential from the emitter bias voltage. A detailed study of the behaviour has not yet been done.

In addition to the already known poloidal mode number $m=1$ of CNT's single mode iondriven oscillations [21], the toroidal mode number is now known: $n=0$. The $m=1, n=0$ structure of this mode is identical to that of the diocotron mode seen in pure toroidal traps [30]. Whereas this mode structure is expected for a pure toroidal field trap, neither the $m=1$ result, nor the new $n=0$ result were expected in CNT. In CNT $n=0$ implies that the poloidal magnetic flux is not conserved by the plasma traveling with the ion-driven mode, and is therefore violating the frozen-in flux condition well known from ideal MHD theory. We hypothesize that both observations can be understood if the electron pressure is highly anisotropic. The anisotropic tensor would lead to force terms, some along B, usually neglected both in the electron fluid equation and the ideal MHD Ohm's law. Numerical simulations show that $>50 \%$ of the electrons are on magnetically trapped orbits, and that there are complicated loss structures in phase space in CNT [31], also consistent with experimental findings [17,32]. The dominant collisional process in these plasmas is electron-neutral collisions, and they are quite rare [17] $\left(\nu_{\mathrm{en}} \approx 500 \mathrm{~s}^{-1}\right.$ for $\left.\eta=0.3\right)$. Therefore, these plasmas are rather collisionless. It is therefore not unreasonable to assume that the electron pressure tensor is anisotropic. 


\section{Summary}

This paper reports the first experimental characterization of the physics of plasmas as the degree of neutrality $(\eta)$ is varied continuously between the two extremes of quasi-neutral and pure electron plasma. The physics of the detected fluctuations is completely different as $\eta$ is varied. The oscillations in CNT's quasi-neutral plasmas have many characteristics in common with instabilities observed in other low- $\beta$ quasi-neutral plasmas confined in toroidal magnetic configurations. The resonant $m=3$ low frequency flute instability observed for $\eta \lesssim 10^{-4}$ is well aligned with the magnetic structure, and is believed to be driven by density gradients (drift waves). However, the physics of the mode detected in electron-rich plasmas with a small but non-negligible amount of ions $(0.03<\eta<0.8)$ is dominated by electrostatics. The mode presents a non-resonant $m=1, n=0$ spatial structure, and the mechanism for instability seems to be identical to the ion-resonant mode observed in Penning traps [33], and pure toroidal traps [30]. Between these two extremes of the neutralization scale, the plasma presents broadband behaviour.

\section{Acknowledgments}

The authors thank Prof. A. H. Boozer, Dr. P. W. Brenner, and Dr. B. Durand de Gevigney for valuable discussions, and Prof. M. E. Mauel for use of the high speed camera. This work was supported by the National Science Foundation, the Department of Energy of the United States of America (Grant Nos. NSF-PHY-04-49813 and NSF-PHY-0903754), and the European Communities under the contract of Association between EURATOM and IPP.

\section{References}

[1] Davidson R C 2001 Physics of Nonneutral Plasmas 2nd ed (Imperial College Press and World Scientific Publishing)

[2] Malmberg J H and deGrassie J S 1975 Phys. Rev. Lett. 35(9) 577-580

[3] Brillouin L 1945 Phys. Rev. 67 260-266

[4] Boozer A H 2005 Phys. Plasmas 12104502 (pages 3)

[5] Zaveri P et al. 1992 Phys. Rev. Lett. 68(22) 3295-3298

[6] Stoneking M R et al. 2002 Phys. Plasmas 9 766-771 ISSN 1070664X

[7] Fasoli A et al. 2010 Plasma Physics and Controlled Fusion 52124020

[8] Pedersen T S and Boozer A H 2002 Phys. Rev. Lett. 88(20) 205002

[9] Pedersen T S et al. 2004 Phys. Plasmas 11 2377-2381 ISSN 1070664X

[10] Pedersen T S et al. 2006 Fusion Sci. Technol. 50 372-381

[11] Boozer A H 1998 Phys. Plasmas 5 1647-1655 ISSN 1070664X

[12] Pedersen T S et al. 2004 Fusion Sci. Technol. 46 200-208

[13] Kremer J P et al. 2006 Phys. Rev. Lett. 97(9) 095003

[14] Kremer J P et al. 2007 Rev. Sci. Instrum. 78013503 ISSN 00346748

[15] Pedersen T S et al. 2008 Plasma and Fusion Research 3 S1022

[16] Brenner P W and Pedersen T S 2012 Phys. Plasmas 19050701 (pages 4)

[17] Berkery J W et al. 2007 Phys. Plasmas 14062503 ISSN 1070664X

[18] Berkery J W et al. 2007 Phys. Plasmas 14084505 ISSN 1070664X

[19] Boozer A H 2005 Rev. Mod. Phys. 76 1071-1141

[20] Lefrancois R G et al. 2005 Phys. Plasmas 12072105 ISSN 1070664X

[21] Marksteiner Q R et al. 2008 Phys. Rev. Lett. 100(6) 065002

[22] Ye M Y and Takamura S 2000 Phys. Plasmas 7 3457-3463

[23] Hutchinson I H 2002 Principles of Plasma Diagnostics 2nd ed (Cambridge University Press)

[24] Marksteiner Q R 2008 Studies of Non-neutral Ion Electron Plasmas Confined on Magnetic Surfaces Ph.D. thesis Columbia University

[25] Brochard F et al. 2005 Phys. Plasmas 12062104 (pages 7)

[26] Grulke O et al. 1999 Phys. Plasmas 6 788-796

[27] Jassby D L 1972 Phys. Fluids 15 1590-1604

[28] Huang N E et al. 1998 Proc. R. Soc. Lond. A $454903-995$

[29] Brenner P W et al. 2008 Plasma Science, IEEE Transactions on 36 1108-1109 ISSN 0093-3813

[30] Stoneking M R et al. 2003 AIP Conference Proceedings 692 310-319 
[31] de Gevigney B D et al. 2009 Phys. Plasmas 16122502 (pages 9)

[32] Brenner P W et al. 2010 Contrib. Plasma Phys. 50 678-682

[33] Davidson R C and Uhm H S 1978 Phys. Fluids 21 60-71 